\section{Decreased mental health care utilization following a psychosocial intervention in caregivers of hematopoietic stem cell transplant patients}

\author{
Rita Ouseph,1,2 Calvin Croy,2 \\ Crystal Natvig,2 Teresa Simoneau,3 \\ Mark L. Laudenslager² \\ 1Duke University, Durham, NC; \\ 2Anschutz Medical Campus, University \\ of Colorado, Denver, CO; \\ 3Presbyterian/St Luke's Medical Center, \\ Denver, CO, USA
}

\section{Abstract}

Caregivers are known to experience increased morbidity when compared to noncaregivers. Does an intervention targeting caregiver distress affect their health care utilization? One hundred forty-eight caregivers of allogeneic hematopoietic stem cell transplant patients were randomized to treatment as usual (TAU) or a psychoeducation, paced respiration, and relaxation (PEPRR) intervention. Assessments of caregivers' service utilization were collected at baseline and 1,3 , and 6 months post-transplant. During the first 30 days after patient transplant, caregiver medical and mental health professional service use decreased while support group attendance peaked. Mixed model regressions showed a significant decrease in mental health service use by the PEPRR group $(\mathrm{P}=0.001)$. At six months caregivers in TAU had predicted marginal probabilities of mental health services utilization over 10 times as high as caregivers in PEPRR (18.1\% vs 1.5\%). Groups failed to differ in medical service $(\mathrm{P}=0.861)$ or support group $(\mathrm{P}=0.067)$ use. We can conclude that participation in PEPRR compared to TAU was associated with reduced mental health service utilization. Caregiver psychosocial support services are critical to improve caregiver outcomes.

\section{Introduction}

As advances in medicine improve patient outcomes, patients' loved ones/caregivers must meet new challenges that can be simultaneously physical, emotional, and spiritual in nature. An allogeneic hematopoietic stem cell transplant is one such medical advance, in that it represents not only treatment but potential cure for otherwise fatal malignancies. Yet it carries life-threatening risks that include graft versus host disease, ${ }^{1}$ infection, ${ }^{2}$ relapse of primary cancer, ${ }^{3}$ and secondary malignancy. 4,5 It also requires a caregiver to be available $24 / 7$ for at least the first 100 days post-transplant. Family members, often a spouse, usually assume this role, which is actually many roles, including: patient advocate, liaison between the medical team and other family members, nursing aide, and coach. The average family member is poorly prepared for this challenge. For these caregivers, mental health is a huge concern. ${ }^{6}$ A number of studies report greater rates of mood and anxiety problems among this population.7,8 A recent meta-analysis of 144 studies found that anxiety in both the caregiver and patients were persistent problems compared to healthy controls. ${ }^{9}$ Among cancer caregivers, $13 \%$ have been found to fit a psychiatric diagnosis, with the most common being panic disorder. Despite this prevalence, less than half of caregivers with a diagnosable disorder receive psychiatric care. 10 This represents a significant gap in mental health care.

However, a new and growing body of literature is finding that patients and caregivers may benefit from the illness experience, a phenomenon popularly described as benefit finding. ${ }^{11}$ Benefit finding refers to the personal growth that can arise out of meeting the many new challenges that come with a diagnosis of severe illness like cancer. Studies have utilized disparate scales to describe this phenomenon. For example, Bower and Segerstrom describe four types of benefit finding:12 an accepting attitude toward life, positive change in selfview, change in interpersonal relationships, and a deeper sense of purpose in life with a greater focus on important goals and priorities. These changes are intimately related to mental health, but not all in the same direction. Kim et al. ${ }^{11}$ found that benefit finding in the domains of empathy and reprioritizing one's life related to greater depressive symptoms, whereas greater acceptance and appreciation of one's life related to less depressive symptoms. Moreover, greater benefit finding was highly associated with the presence of social support for the caregiver. This highlights the promising potential of caregiver interventions for improving caregiver morbidity, which, despite benefit finding, remains higher than in non-caregiver populations.

The increased morbidity found in caregivers can be thought of as mediated by three factors: $13-15$ i) the stress of caregiving leading to disrupted immune and neuroendocrine regulation, 16 ii) impaired self-care behaviors (e.g. nutrition, exercise, adequate sleep, abstinence from tobacco and alcohol), and/or iii) reduced health care utilization, with bi-directional influences among these factors. While the first two factors have been described at length, less is known about the third: caregivers' mental and physical health care utilization. Several
Correspondence: Rita Ouseph, Anschutz Medical Campus, University of Colorado, 12700 East $19^{\text {th }}$ Place, Room 3410A, Building P15, Aurora, CO 80045, USA. Tel. +1.817.247.4793.

E-mail: rita.ouseph@gmail.com

Key words: mental health, caregivers, stress, intervention.

Acknowledgments: we would like to thank all the caregivers and patients who participated in this study. We also thank Kristin Kilbourn, Susan Lutgendorf, and Anne Libby, for help in study concept and design, Janet Spradley, for conduction of the intervention, Samuel Phillips, for acquisition of data, and Andrew Krystal, for mentorship.

Contributions: ML, TS, study concept and design; CC, ML, CN, RO, TS, acquisition, analysis, and interpretation of data; CC, ML, CN, RO, TS, drafting of the manuscript or critical revision for important intellectual content; ML, CN, RO, CC, TS, final approval.

Conflict of interests: the authors declare no potential conflict of interests.

Funding: NIH grants CA126971 (ML) and K07 AG030337 Subproject (ML).

Conference presentation: portions of this study were presented as a poster at the University of Colorado Denver Anschutz Campus Department of Psychiatry Junior Faculty Poster Session and an oral presentation at the University of Colorado Denver Anschutz Campus Palliative Care Conference.

Received for publication: 9 October 2013.

Revision received: 4 January 2014

Accepted for publication: 8 January 2014.

This work is licensed under a Creative Commons Attribution NonCommercial 3.0 License (CC BYNC 3.0).

CCCopyright R. Ouseph et al., 2014

Licensee PAGEPress, Italy

Mental Illness 2014; 6:5120

doi:10.4081/mi.2014.5120

studies found equal or increased use of health services by caregivers compared to non-caregivers, $7,17-21$ with persons reporting greater caregiver burden also reporting greater health care use..$^{21}$ Spousal caregivers of patients with dementia used similar amounts of outpatient health services but more Emergency Room services than a comparison group of non-caregiving spouses. ${ }^{22}$ This suggests that the time demands of caregiving may cause caregivers to discontinue scheduled preventive outpatient care and resort to urgent care that is available 24 hours a day, 7 days a week. Further, caregivers with greater depressive symptoms have been shown to use more acute care, ${ }^{23}$ signaling the importance of mental health services 
within the wider health system. Illustrative of this fact, a manualized 8-session intervention for family caregivers of persons with dementia led to less use of outpatient services by those caregivers through improvements in anxiety and depression symptomatology over an 8 month study period there was no net cost difference between the intervention and treatment as usual. 24

The present study explored two questions: i) do allogeneic HSCT caregivers' mental health and medical health services utilization change post-transplant? and ii) would an intervention directed to improved caregiver coping influence their use of medical and behavioral services? We theorized that an eight session individual cognitive and behavioral stress management intervention, ${ }^{25}$ which we called psychoeducation, paced respiration, and relaxation (PEPRR), would prompt caregivers to improve self-care and thereby seek more medical services. Additionally, we hypothesized that caregivers in PEPRR would learn enhanced behavioral coping skills and thereby require less traditional mental health services, either as support groups or one-on-one meetings with a mental health professional.

\section{Materials and Methods}

\section{Study design}

This was a longitudinal randomized control trial with intent to treat. Patient/caregiver dyads were recruited consecutively from a HSCT program in Colorado. Subjects were given information about the study during the caregiver and patient psychosocial assessment, a standard requirement for a HSCT. All research subjects provided informed consent and the study was approved by the Colorado Multi-Institutional Review Board. A total of 148 participating dyads were consented and randomized by permuted block design to either PEPRR or treatment as usual (TAU) prior to transplant, with the first scheduled intervention occurring on average 16 days post-transplant. Caregiver demographics are described in Table 1. There were no statistically significant differences (using $\mathrm{P} \leq 0.05$ ) in these demographic characteristics between PEPRR or TAU.

Caregiver inclusion criteria were: i) primary caregiver for an allogeneic HSCT patient for at least $50 \%$ of the time during the first 100 days post-transplant, ii) speak and read English, iii) 18 years or older, and iv) access to a telephone. Exclusion criteria included: i) history of any psychiatric disorder in the past 18 months unrelated to the patient's illness, ii) any medical condition likely to influence neuroendocrine or immune markers (to be reported elsewhere), iii) self-reported alcohol consumption greater than 2 drinks/day, or iv) use of any steroid medications.

\section{Intervention}

PEPRR consisted of eight one-on-one sessions ( 4 weekly 60 minute sessions followed by 4 biweekly 60 minute sessions) with a social worker provided during the first 100 days posttransplant. Most sessions occurred at the outpatient HSCT clinic, so they coincided with the patients' transplant follow-up visits with the oncologist, thereby providing ease of scheduling. Because the social worker was located at the transplant clinic, she could also facilitate communication between the caregiver and the patient's physician.
The PEPRR intervention was modified from an effective cognitive-behavioral stress management program for early stage breast cancer patients. ${ }^{26}$ Topics contained an emphasis on self-care and included education about stress, coping skills, and problem solving techniques, as well as identifying helpful resources in the community. A biofeedback device, the RESPeRATE, 27 was utilized to help entrain slowing of respiration. TAU caregivers were encouraged to voluntarily take advantage of the many free resources and services available at the transplant center (support groups, individual counseling, and education) but did not receive the scheduled intervention sessions nor training with the RESPeRATE. PEPRR caregivers were also encouraged to utilize these free resources as well.

\section{Assessment}

Caregivers in PEPRR and TAU completed survey questionnaires at 4 time points: baseline (prior to transplant and randomization) and 1, 3, and 6 months post-transplant. All times were anchored to the day of transplant as day 0. At each assessment, caregivers answered questions about their utilization of health services. The first outcome variable, support group attendance, reflected the response to the question: During the past 4 weeks, did you attend any support groups? The second outcome variable, medical service use, was: During the past 4 weeks, did you have an office visit with a physicianphysician's assistant, or nurse/nurse practitioner for any medical problems? Our third outcome variable, mental health service use, was defined by examining the caregiver's responses to two questions: i) During the past 4 weeks, were you seen by a psychiatrist, psychologist, social

Table 1. Caregiver demographics.

\begin{tabular}{|c|c|c|}
\hline Caregiver characteristics $(n=74)$ & TAU, No. of caregiver (\%) & PEPRR, No. of caregiver $(\%)$ \\
\hline Age mean (SD) & $54.8(12.4)$ & $52.2(12.2)$ \\
\hline Range (median), y & $24-80(55.0)$ & $21-77(54.0)$ \\
\hline \multicolumn{3}{|l|}{ Sex } \\
\hline Female & $56(75.7)$ & $56(75.7)$ \\
\hline Male & $17(23.0)$ & $18(24.3)$ \\
\hline \multicolumn{3}{|l|}{ Ethnicity } \\
\hline Caucasian & $68(91.9)$ & $65(87.8)$ \\
\hline Other & $4(5.5)$ & $8(10.9)$ \\
\hline \multicolumn{3}{|l|}{ Annual individual income, US Dollars } \\
\hline$<25,000$ & $13(17.6)$ & $11(14.9)$ \\
\hline $25,000-44,999$ & $15(20.3)$ & $14(18.9)$ \\
\hline $45,000-64,999$ & $11(14.9)$ & $15(20.3)$ \\
\hline$>65,000$ & $31(41.9)$ & $29(39.2)$ \\
\hline \multicolumn{3}{|l|}{ Relationship to patient } \\
\hline Spouse/partner & $46(62.2)$ & $57(77.0)$ \\
\hline Parent & $19(25.7)$ & $8(10.8)$ \\
\hline Other & $8(10.8)$ & $8(10.8)$ \\
\hline
\end{tabular}


worker, counselor or therapist? and i) Was this a scheduled study session? Our outcome variable for use of mental health service by participants of PEPRR was coded yes if the caregiver answered yes to question 1 and no to question 2 ; and coded no if the caregiver answered no to question $10 \mathrm{OR}$ answered yes to both questions 1 and 2 (treating positive responses to question 1 that may have included study sessions as indicating no external service use). For participants in TAU, question 2 was not relevant as they did not participate in any study sessions. For these participants, the outcome variable was coded identical to the answer to question 1.

\section{Statistical analysis}

We first computed the proportions of caregivers who exhibited any outcome behavior at each assessment time. Next, we determined whether type of treatment (PEPRR vs TAU) had an effect on the probability of service utilization by constructing random-intercept random-coefficient mixed logistic regressions. ${ }^{28}$ Mixed logistic regression was used to account for correlations among outcome values across time for each caregiver. Models that don't take such longitudinal correlations into account yield standard errors that are too small and thus can produce spuriously significant findings. Describing our logistic regressions using multilevel terminology, at Level 1 each dichotomous outcome was regressed on the number of months since transplant. At Level 2, both the Level 1 intercept and the coefficient for months were predicted by whether or not the outcome occurred at baseline (prior to intervention) and by type of treatment received. Therefore we examined treatment effect in terms of predicted usage probabilities while controlling for baseline differences. All models were calculated using SAS Proc NLMIXED (SAS version 9.3; SAS Institute, 2010). Outcome occurrences were modeled from 1 month post-transplant to 6 months post-transplant. To test whether treatment affected outcomes, we employed the deviance difference test. We compared fits of each model, quantified as its-2loglikelihood or the deviance, with treatment included as a predictor variable to the fit of the model with treatment omitted. If the $p$ value of the difference in the deviances of the two models was significant $(\mathrm{P} \leq 0.05)$, we then analyzed models to test whether the effect of treatment operated through the Level 1 random intercept or the Level 1 random coefficient on elapsed months. We used this approach for assessing the significance of treatment effect rather than dividing the treatment coefficient by its standard error, known as a Wald test. This was because regression standard errors are known to be overestimated in certain conditions. ${ }^{27}$

The proportions of participants utilizing mental health and support group services displayed curvilinear trajectories across time. We therefore attempted to fit logistic regressions using dichotomous indicators for the 3 and 6 month time values and also tried fitting quadratic logistic regressions to these trajectories. Since these models either failed to converge (attend support group) or did not fit the data any better than a linear logistic regression (mental health service use, deviance difference test $\mathrm{P}=0.809$ ), our final logistic regressions were linear for all three outcome events.

\section{Results}

The present analysis suggested that participation in the PEPRR intervention satisfied or reduced the need for specific mental health services in those caregivers compared to TAU. All modeling results show the effects of treatment type after controlling for service use prior to their patient's transplant. Figure 1 shows study flow and caregiver response rates at different stages. In Figure 2, the A and C panels represent observed rates of service utilization. The B and D panels represent corresponding rates of service utilization predicted by the model for an average study participant (variance components for random intercept and random coefficient for time set at mean values of 0 ).

\section{Mental health service use}

Caregivers' visits with a mental health professional exhibited a decline from baseline to one month after transplant, followed by a plateau as indicated in Figure 2A. Inclusion of treatment in the mixed logistic regression significantly improved the fit (deviance difference test $\mathrm{P}=0.001$ ). We further found that treatment did not have a significant effect on the intercept of the logistic regression (deviance difference test $\mathrm{P}=0.65$ ), supporting that the PEPRR and TAU effects were not significantly different at 0 months (time of transplant). However, treatment did have an effect on the coefficient for time that approached statistical significance (deviance difference test $\mathrm{P}=0.10$ ), suggesting that the proportion of caregivers in PEPRR who reported seeing an outside mental health professional declined at

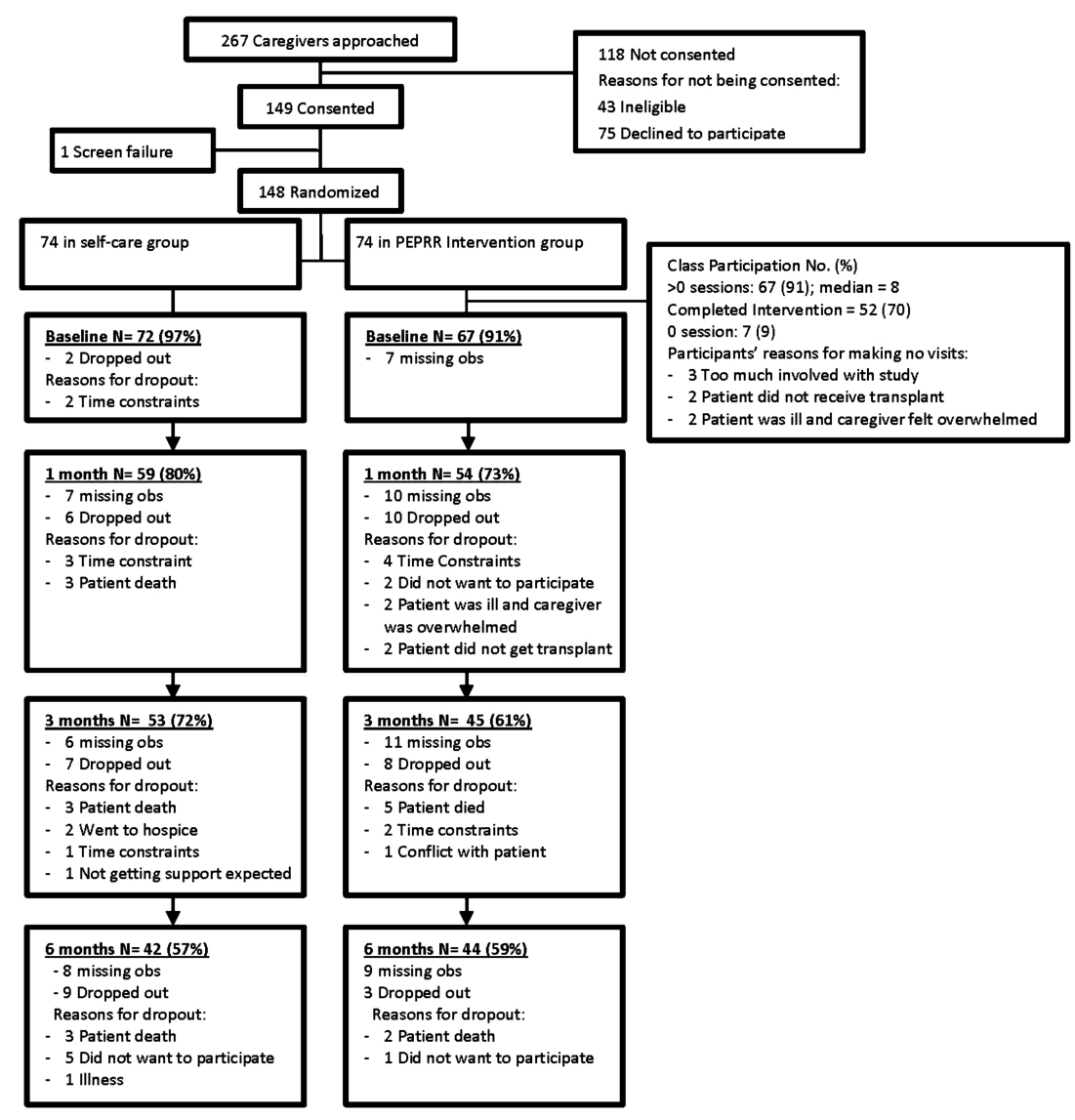

Figure 1. Caregiver response rates and flow through the study. 


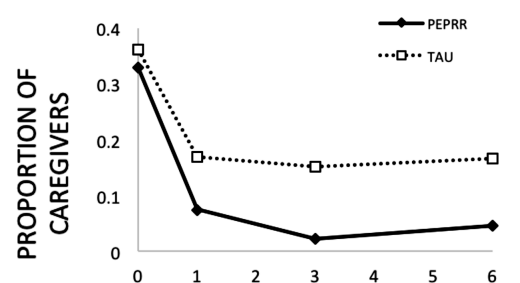

A MONTHS AFTER TRANSPLANT

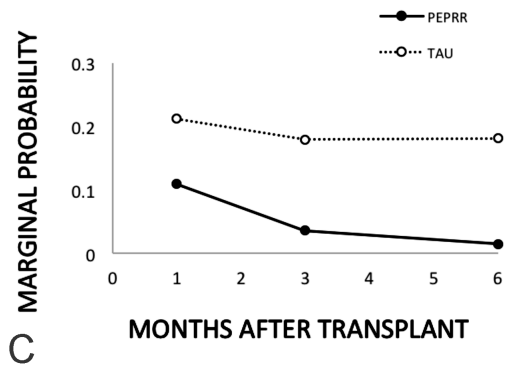

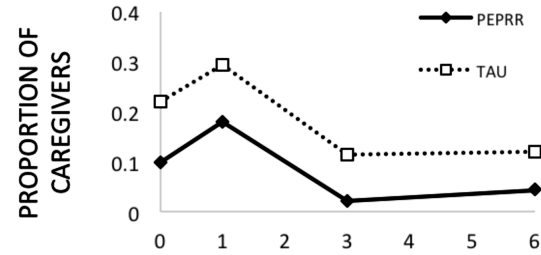

B MONTHS AFTER TRANSPLANT

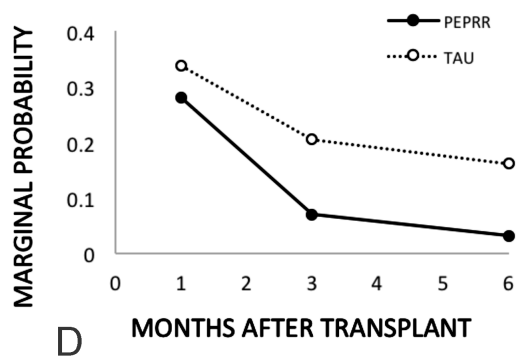

Figure 2. A) shows the proportions of caregivers who reported visiting a mental health professional at each time point, through 6 months. C) shows the predicted marginal probabilities of mental health professional utilization at each time point. B) shows the proportions of caregivers who reported attending a support group at each time point, through 6 months. D) shows the predicted marginal probabilities predicting support group utilization at each time point.

a faster rate than the proportion of caregivers in TAU.

Marginal predicted probabilities of seeking a mental health professional for caregivers in PEPRR and caregivers in TAU based on population-averaged analyses are depicted in Figure 2C. The marginal probability at one month for a caregiver in PEPRR is half that for a caregiver in TAU (10.9\% vs $21.2 \%)$. At six months caregivers in TAU had marginal probabilities over 10 times as high as caregivers in PEPRR (18.1\% vs $1.5 \%)$.

\section{Medical health service use}

Medical health service utilization decreased from baseline to one month, and increased thereafter until month six. However, the mixed logistic regression (which controlled for differences in service use at baseline) failed to show a statistically significant effect of treatment group on medical service use trajectories (deviance difference test, $\mathrm{P}=0.86$ ).

\section{Support group use}

Interestingly, caregivers assigned to TAU exhibited statistically significant greater use of outside support group utilization at baseline (22.06\% in TAU vs 9.86\% in PEPRR, chi-square $\mathrm{P}=0.049$ ). The present model controlled for this baseline disparity. At one month there was an increase in support group attendance as shown in Figure 2B, but we found no evidence that the treatment groups differed in their rates of service use at this time point (chi-square $\mathrm{P}=0.147$ ).
Attendance tended to drop from 1 to 6 months for both treatment types; mixed logistic regression suggests that decline in use was steeper for the PEPRR treatment group (deviance difference test, $\mathrm{P}=0.067)$. In an additional analysis, we found a significant effect of treatment on the coefficient for months (deviance difference test $\mathrm{P}=0.038$ ). Marginal probabilities of support group attendance from our model are depicted in Figure 2D.

\section{Discussion}

These findings show that during the month after transplant visits to mental health and medical service providers declined, yet attendance at support groups increased. There could be several reasons for these patterns. Because patients are typically still hospitalized during the 30 days post-transplant, caregivers may have had time available to attend support groups, which are typically held at fixed times rather than scheduled at the participants' convenience. Thus after patient discharge the caregiver's time becomes less flexible. Additionally, the transplant program offered a weekly caregiver support group on the inpatient HSCT floor which caregivers may have taken advantage of because of the convenience. Regrettably, the specifics of support group attendance was not determined. Caregivers may have seen support groups as a source of information as well as emotional support from experienced caregivers that would help prepare them for imminent challenges. During this period, perceived stress is high as caregivers deal with the uncertainty of the patient's outcome, still precarious after a risky medical procedure, and also prepare for full-time home caregiving. Visiting a personal mental health or medical professional may have been displaced by a need for support connected to their immediate experience.

During the 3-6 months post-transplant when caregiving duties begin to decline beyond the initial 100 day HSCT caregiver requirement, medical service utilization increased while mental health service use (support groups and individual visits) decreased in both groups. This matches what is seen in the literature, as caregivers tend to show increased use of medical care when caregiving duties subside. ${ }^{29}$ The decrease in mental healthcare may also reflect decreased caregiver need for this sort of support. Given that psychiatric illness unrelated to caregiving duties in the past 18 months was an exclusion criterion for study participation, caregiver stress in this study was mostly situational. Therefore, when caregiving duties (i.e., the root of the situational stress) abated, HSCT caregiver need for mental health care also lessened.

PEPRR influenced caregiver use of mental health services as originally hypothesized. The prediction was that PEPRR might be associated with less use of mental health services due to increased coping skills. Use of mental health professional services in all caregivers declined after 1 month post-transplant, with the PEPRR group showing a steeper decline. The fact that perceived stress also declined during this time period for caregivers in PEPRR (and not for caregivers in TAU) ${ }^{25}$ suggests that the skills learned in PEPRR mitigated the need for these services. PEPRR was designed to assist caregivers specifically in acknowledging the extreme life changes after a transplant, adapting to these challenges as much as possible, and ultimately, hopefully, appreciating the value within the caregiver role. As such, PEPRR may have improved caregiver mental health by promoting benefit finding within this population.

\section{Conclusions}

This study has several limitations. Several mediators found in the literature to influence mental health care utilization were not assessed in our study. 30 While the PEPRR and TAU cohorts showed no statistical differences in major demographic variables, we cannot exclude the possibility that unmeasured factors, e.g. religion and historical contact with the mental health care system prior to trans- 
plantation, may have contributed to differential use of health care. In addition, our study assessed caregivers up to six months posttransplant. Future research will look at whether differences in health care utilization persist over a longer period of time. It also only considered data on outpatient health care utilization. Future research should also gather cost data and use electronic medical records to get a global perspective of this intervention's impact on health care for both patient and caregiver. Also, future questionnaires will use more precise language so participants understand they are reporting care separate from the study itself. Finally, participants in TAU, while able to utilize free psychological services of their own volition, did not receive an active intervention. Therefore, the decrease in mental health care utilization may not be attributable specifically to PEPRR but rather to all active interventions. Future research should examine intervention qualities more closely to parse out the most effective strategies for stress reduction.

In conclusion, the decrease in both perceived stress and mental health service utilization indicates that PEPRR may be a worthwhile addition to transplant program services. Although transplant programs offer caregiver support services (support groups, social workers, and psychologists), alternative methods like PEPRR can help since few caregivers voluntarily avail themselves of these traditional services and support groups alone may not impact stress levels. We thus propose that PEPRR is a cost-effective, feasible manner to help provide improved mental health care for caregivers of allogeneic HSCT patients.

\section{References}

1. Copelan EA. Hematopoietic stem-cell transplantation. New Engl J Med 2006;354: 1813-26.

2. Neofytos D, Horn D, Anaissie E, et al. Epidemiology and outcome of invasive fungal infection in adult hematopoietic stem cell transplant recipients: analysis of Multicenter Prospective Antifungal Therapy (PATH) alliance registry. Clin Infect Dis 2009;48:265-73.

3. Martinelli G, Terragna C, Zamagni E, et al. Molecular remission after allogeneic or autologous transplantation of hematopoietic stem cells for multiple myeloma. J Clin Oncol 2000;18:2273-81.

4. Majhail NS, Brazauskas R, Rizzo JD, et al.
Secondary solid cancers after allogeneic hematopoietic cell transplantation using busulfan-cyclophosphamide conditioning. Blood 2011;117:316-22.

5. Lowe T, Bhatia S, Somlo G. Second malignancies after allogeneic hematopoietic cell transplantation. Biol Blood Marrow Transplan 2007;13:1121-34.

6. Laudenslager M. Anatomy of an Illness: control from a caregiver's perspective. Brain Behav Immun 2014;36:1-8.

7. Moore MJ, Zhu CW, Clipp EC. Informal costs of dementia care: estimates from the national longitudinal caregiver study. J Gerontol B Psychol Sci Soc Sci 2001;56: S219-28.

8. Simoneau TL, Mikulich-Gilbertson SK, Natvig C, et al. Elevated peri-transplant distress in caregivers of allogeneic blood or marrow transplant patients. Psychooncology 2013;22:2064-70.

9. Mitchell AJ, Ferguson DW, Gill J, et al. Depression and anxiety in long-term cancer survivors compared with spouses and healthy controls: a systematic review and meta-analysis. Lancet Oncol 2013;14:72132.

10. Vanderwerker LC, Laff RE, Kadan-Lottick NS, et al. Psychiatric disorders and mental health service use among caregivers of advanced cancer patients. J Clin Oncol 2005;23:6899-907.

11. Kim Y, Schulz R, Carver CS. Benefit-finding in the cancer caregiving experience. Psychosom Med 2007;69:283-91.

12. Bower JE, Segerstrom SC. Stress management, finding benefit, and immune function: positive mechanisms for intervention effects on physiology. J Psychosom Res 2004;56:9-11.

13. Rohleder N, Marin TJ, Ma R, Miller GE. Biologic cost of caring for a cancer patient: dysregulation of pro- and anti-inflammatory signaling pathways. J Clin Oncol 2009; 27:2909-15.

14. Lutgendorf SK, Laudenslager ML. Care of the caregiver: stress and dysregulation of inflammatory control in cancer caregivers. J Clin Oncol 2009;27:2894-5.

15. Christakis NA, Allison PD. Mortality after the hospitalization of a spouse. New Engl J Med 2006;354:719-30.

16. Gouin JP, Hantsoo L, Kiecolt-Glaser JK. Immune dysregulation and chronic stress among older adults: a review. Neuroimmunomodulation 2008;15:251-9.

17. Scharlach AE, Midanik LT, Runkle MC, Soghikian K. Health practices of adults with elder care responsibilities. Prev Med
1997;26:155-61.

18. Fillit HM. The pharmacoeconomics of Alzheimer's disease. Am J Manag Care 2000;6:S1139-44.

19. Vitaliano PP, Zhang J, Scanlan JM. Is caregiving hazardous to one's physical health? A meta-analysis. Psychologic Bull 2003; 129:946-72.

20. Burns A. The burden of Alzheimer's disease. Int J Neuropsychopharmacol 2000; 3:31-8.

21. Son J, Erno A, Shea DG, et al. The caregiver stress process and health outcomes. J Aging Health 2007;19:871-87.

22. Kolanowski AM, Fick D, Waller JL, Shea D. Spouses of persons with dementia: their healthcare problems, utilization, and costs. Res Nurs Health 2004;27:296-306.

23. Schubert CC, Boustani M, Callahan CM, et al. Acute care utilization by dementia caregivers within urban primary care practices. J General Intern Med 2008;23:173640.

24. Knapp M, King D, Romeo R, et al. Cost effectiveness of a manual based coping strategy programme in promoting the mental health of family carers of people with dementia (the START (STrAtegies for RelaTives) study): a pragmatic randomised controlled trial. BMJ 2013;347: 6342 .

25. Laudenslager ML, Natvig C. Challenges to conducting a randomized control behavioral intervention trial for caregivers of hematopoietic stem cell transplant recipients. [In press].

26. Antoni MH, Wimberly SR, Lechner SC, et al. Reduction of cancer-specific thought intrusions and anxiety symptoms with a stress management intervention among women undergoing treatment for breast cancer. Am J Psychiatry 2006;163:1791-7.

27. Mahtani KR, Nunan D, Heneghan CJ. Device-guided breathing exercises in the control of human blood pressure: systematic review and meta-analysis. J Hypertension 2012;30:852-60.

28. Hox J. Multilevel analysis: techniques and applications. 2 ed. New York: Routledge; 2010.

29. Grasel E. When home care ends - changes in the physical health of informal caregivers caring for dementia patients: a longitudinal study. J Am Geriatr Soc 2002;50: 843-9.

30. Ng TP, Nyunt MS, Chiam PC, Kua EH. Religion, health beliefs and the use of mental health services by the elderly. Aging Mental Health 2011;15:143-9. 